\title{
AGGRESSIVE SURGICAL MANAGEMENT IN LOCALIZED PULMONARY MYCOTIC AND NONMYCOTIC INFECTIONS FOR NEUTROPENIC PATIENTS WITH ACUTE LEUKEMIIA: REPORT OF EIGHTEEN CASES
}

O. Baron, $\mathrm{MD}^{\mathrm{a}}$

B. Guillaumé, $\mathrm{MD}^{\mathrm{b}}$

P. Moreau, $\mathrm{MD}^{\mathrm{c}}$

P. Germaud, $\mathrm{MD}^{\mathrm{b}}$

P. Despins, MD

A. Y. De Lajartre, $\mathrm{MD}^{\mathrm{d}}$

J. L. Michaud, MD

Sponsor:

Willard A. Fry, MD
Objective: To prevent hemoptysis and relapse during subsequent chemotherapy-induced neutropenia in patients with localized forms of invasive pulmonary aspergillosis, we adopted an aggressive surgical approach. Methods: From 1988 to 1996,18 patients with hematologic diseases were referred with the diagnosis of localized invasive pulmonary aspergillosis. The diagnosis was based on clinical features, failure to respond to antibiotic therapy, an air crescent sign suggestive of aspergillosis on the computed tomographic scan $(39 \%)$, and retrieval of fungi by bronchoalveolar lavage $(44 \%)$. Results: The following procedures were done: one pneumonectomy, four bilobectomies, seven lobectomies, six wedge resections, and one lobectomy with wedge resection (one patient had two procedures). No perioperative deaths or complications occurred. The histologic examination confirmed the diagnosis of invasive pulmonary aspergillosis in $\mathbf{1 2}$ patients. The six other diagnoses were as follows: one case of classic aspergilloma, one case of pneumonia, and four cases of pulmonary abscess. According to univariate analysis, thoracic pain was less common in the group with noninvasive pulmonary aspergillosis (1/6) than in the group with invasive pulmonary aspergillosis $(8 / 12)(p<0.05)$. Sixteen patients required subsequent hematologic treatments. Sixty-six percent of the patients are alive with a mean follow-up of $29.1 \pm 27.8$ months (range 2 to 103 months), with no statistically significant difference between the invasive and the noninvasive pulmonary aspergillosis groups. Five patients died of a recurrence of their malignant disease at a mean of $17.2 \pm 12.5$ months (range 2 to 30 months), and one had a cerebral recurrence of Aspergillus infection during a bone marrow transplantation 3 months later. Conclusion: Aggressive surgical management radically improves the prognosis of invasive pulmonary aspergillosis, even if the surgical indications include some nonmycotic infections because of the difficulty in establishing the clinical diagnosis. (J Thorac Cardiovasc Surg 1998;115:63-9)
From the Department of Cardiothoracic Surgery, ${ }^{a}$ the Department of Pneumology, ${ }^{\mathrm{b}}$ the Department of Hematology, ${ }^{\mathrm{c}}$ and the Department of Pathology, ${ }^{\mathrm{d}}$ Nantes University Center Hospital, Nantes, France.

Read at the Seventy-seventh Annual Meeting of The American Association for Thoracic Surgery, Washington, D.C., May 4-7, 1997.

Received for publication May 7, 1997; revisions requested June 2, 1997; revisions received August 1, 1997; accepted for publication August 4, 1997.

Address for reprints: J. L. Michaud, MD, Department of Cardiothoracic Surgery, Hôpital Guillaume et René Laënnec, BP 1005, 44035 Nantes, France.

Copyright (C) 1998 by Mosby, Inc.

$0022-5223 / 98 \$ 5.00+0 \quad \mathbf{1 2 / 6 / 8 5 2 7 7}$
Datients with hematologic malignant diseases necessitating high-dose chemotherapy or even bone marrow transplantation are at risk for numerous infectious complications. ${ }^{1,2}$ The respiratory system is particularly susceptible to such infections. Among the most devastating infections are the pulmonary mycoses. ${ }^{3}$ Fungal infections in patients with acute leukemia who undergo induction chemotherapy are caused in $58 \%$ of cases by Candida and in $30 \%$ by Aspergillus. ${ }^{4,5}$

The prognosis of invasive pulmonary aspergillosis (IPA) is poor. ${ }^{6}$ However, in selected patients with a localized lesion suspected to be a form of IPA, the optimal mode of treatment remains undefined. The current study reports our experience in the surgical 
Table I. Summary of the patients' symptoms

\begin{tabular}{ccllcc}
\hline Patient No. & Age $(y r)$ & $\begin{array}{c}\text { Hematologic } \\
\text { disease }\end{array}$ & \multicolumn{1}{c}{ Symptoms ${ }^{*}$} & BAL & Histology \\
\hline 1 & 52 & Myeloma & Hemoptysis, chest pain & - & IPA \\
2 & 15 & LAL & Fever, chest pain, hemoptysis & + & IPA \\
3 & 53 & MAL & Fever, chest pain, AC sign & + & IPA \\
4 & 47 & MAL & Fever, hemoptysis, AC sign & - & IPA \\
5 & 48 & Myeloma & Fever, AC sign & + & IPA \\
6 & 74 & MAL & Fever & - & IPA \\
7 & 54 & MAL & Fever, chest pain & + & IPA \\
8 & 40 & MAL & Fever, AC sign & + & IPA \\
9 & 29 & MAL & Fever, chest pain, AC sign & - & IPA \\
10 & 30 & MAL & Fever, chest pain, AC sign & - & IPA \\
11 & 51 & MAL & Fever, chest pain & + & IPA \\
12 & 29 & MAL & Fever, chest pain & - & Abscess \\
13 & 38 & MAL & Fever & - & Abscess \\
14 & 29 & MAL & Fever, chest pain, AC sign & - & Abscess \\
15 & 57 & MAL & Hemoptysis & - & Abscess \\
17 & 26 & LAL & Fever & + & ASP \\
18 & 52 & MAL & Fever & - & Pneumonia \\
\hline
\end{tabular}

$\overline{B A L}$, Bronchoalveolar lavage; $L A L$, lymphoblastic acute leukemia; $M A L$, myeloblastic acute leukemia; $A C$, air crescent; $I P A$, invasive pulmonary aspergillosis; $A S P$, aspergilloma.

*All patients had pneumonia and neutropenia.

management of 18 patients with pulmonary lesions highly suspected to be IPA. Our approach prevented hemoptysis and allowed subsequent cytotoxic treatment with prolonged neutropenia.

\section{Patients and methods}

From 1988 to 1996, in the Department of Hematology in Nantes University Hospital, among 1600 patients who received intensive cytotoxic chemotherapy, 80 cases of IPA were diagnosed (5\%). Surgical resection was considered for patients with a good hematologic response to therapy (e.g., normal peripheral counts after drug-induced aplasia: polymorphonuclear cells $>1500 / \mu \mathrm{l}$ ), normal pulmonary function with a good pulmonary reserve, and strong probability of localized IPA.

Eighteen consecutive patients of the 80 (22.5\%) who met these criteria underwent surgical resection. The patients comprised 10 women and eight men (median 47 years, range 15 to 74 years) who were treated with high-dose chemotherapy for acute myelogenous leukemia $(n=13)$, acute lymphoblastic leukemia $(n=3)$, and multiple myeloma $(n=2)$. The diagnosis of IPA was suspected during aplasia after the first induction of chemotherapy for all patients, except for two who were treated by salvage chemotherapy for relapse. No patient was known to have active fungal or bacterial infection at the time chemotherapy was performed. The median duration of chemotherapy-induced neutropenia (polymorphonuclear cells $<500 / \mu \mathrm{l}$ ) was 33 days (range 16 to 60 days).

The diagnosis (Table I) of IPA was based on clinical features (fever 16/18, chest pain 9/18, hemoptysis 5/18), failure to respond to broad-spectrum antibiotic therapy, the presence of an air crescent sign on the computed tomographic (CT) scan (7/18), and retrieval of fungi by bronchoalveolar lavage (BAL) (8/18). The diagnosis was considered to be almost certain when the BAL results showed abnormalities, and in the 10 other patients the association of the clinical and radiologic signs rendered the diagnosis highly likely. Serologic examination was not routinely used in our series because negativity is common during aplasia. The most common site of these lesions was the upper lobe of the lung (15/18). Five lesions were located in the lower lobe and three in the middle lobe. Five patients had multiple locations, and one had bilateral lesions.

Once the diagnosis of IPA was suspected, 17 patients received an antifungal medical treatment for 32 days (range 12 to 90 days) before surgical treatment. Amphotericin B (1 mg/kg per day) alone was given in eight patients, and in nine other patients it was followed by oral itraconazole (400 mg per day). Itraconazole was never given alone.

The average preoperative polymorphonuclear cell count was $6721 / \mu \mathrm{l}(600$ to $17,000 / \mu \mathrm{l})$ and the average preoperative platelet count was $245,000 / \mu$ l (range 30,000 to $745,000 / \mu l$ ). Only two patients received platelet transfusions before the operation. Results of coagulation studies were within normal limits in all patients. In two patients the operation was performed on an urgent basis because the mass was located close to the main pulmonary artery.

Operative procedures included one pneumonectomy, four bilobectomies, seven lobectomies, six wedge resections, and one lobectomy with wedge resection (one patient had two procedures). Usually the pleural surface was not adherent to the chest wall, but sometimes smooth pleural adhesions were present and were easily dissected. No perioperative or postoperative deaths or complica- 

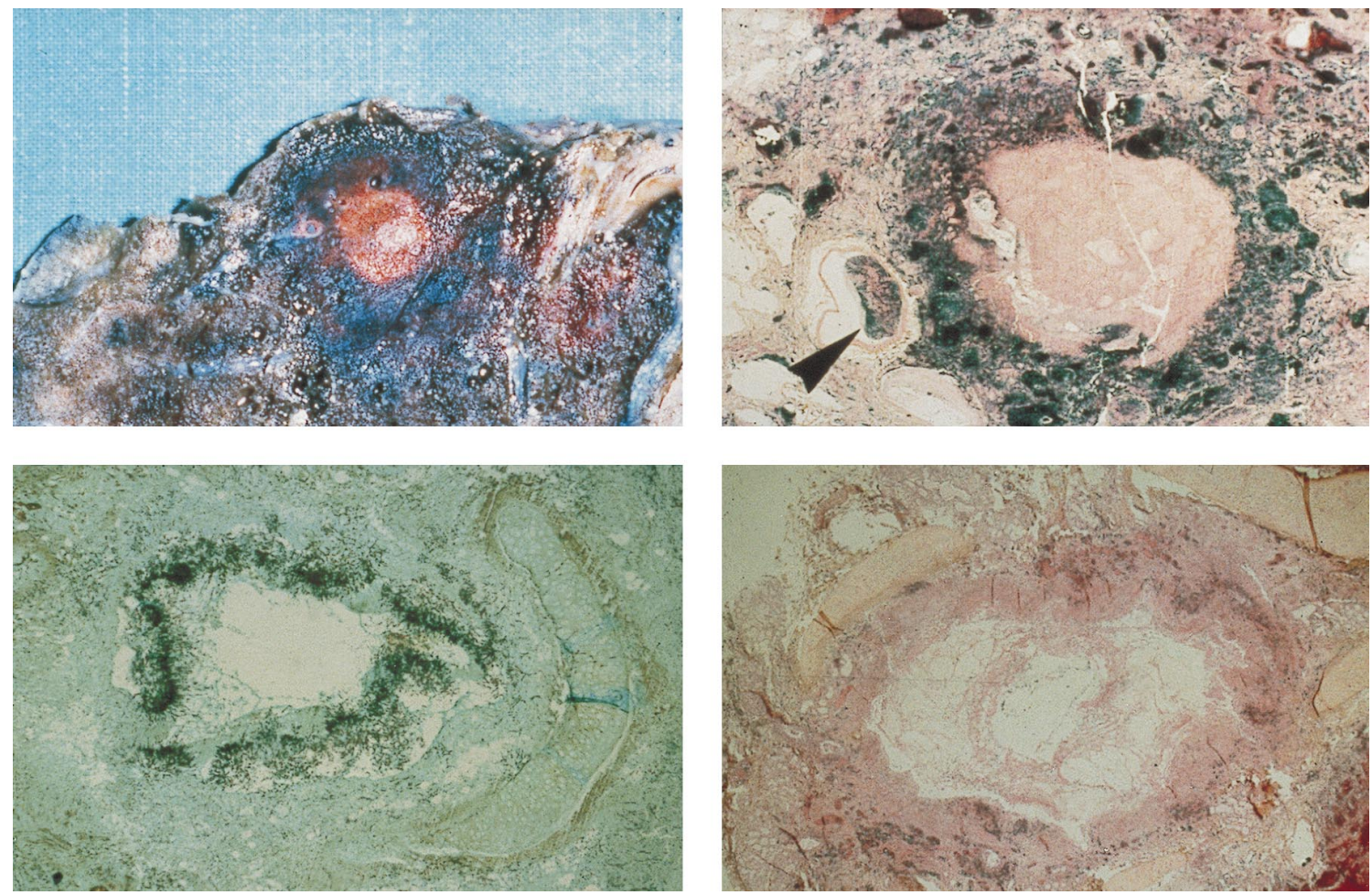

Fig. 1. Typical IPA. Upper left, Macroscopic view of the ball infarction. Lower left, Bronchopulmonary invasion by Aspergillus (Grocott coloration). Lower right, Bronchopulmonary necrosis. Upper right, Blood vessel invasion (arrow) with ensuing thrombosis and infarction surrounded by an acute inflammation.

tions occurred, in particular, no bleeding, no empyema, and no residual space problems.

The histologic examination of the resected specimens confirmed the diagnosis of IPA in 12 patients in whom invasion of blood vessels by the fungus led to ball or triangular pulmonary infarction (Fig. 1). This infarcted piece of tissue was often separated from the surrounding tissues by phagocytes. In the six other patients, the diagnoses were as follows: classic aspergilloma, one case; pneumonia, one case; and pulmonary abscess, four cases, although three of them had Aspergillus colonization without the typical invasion of blood vessels by Aspergillus that defines IPA.

According to univariate analysis, in the group with noninvasive pulmonary aspergillosis (NIPA) fewer patients had thoracic pain $(1 / 6)$ than in the group with IPA $(8 / 12)(p<0.05)$; the likelihood of finding an air crescent sign on the CT scan was less (1/6 in the NIPA group vs 6/12 in the IPA group), as was the likelihood of retrieving aspergillus by BAL (1/6 in the NIPA group vs $7 / 12$ in the IPA group).

Sixteen patients received antifungal therapy after the operation. Sixteen patients required subsequent hematologic treatments: six received antileukemic chemotherapy and 10 received high-dose chemotherapy and total-body irradiation followed by autologous stem cell transplantation. All received prophylactic amphotericin B during additional neutropenia. At a mean follow-up of $29.1 \pm$ 27.8 months (range 2 to 103 months), $66.7 \%$ of the patients are alive (range 2 to 103 months). There is no statistically significant difference between the IPA and the NIPA groups. Five patients died $(27.8 \%)$ of a recurrence of their malignant disease at a mean of $17.2 \pm 12.5$ months (range 2 to 30 months), and one died (5.5\%) of a suspected cerebral recurrence of Aspergillus infection during a bone marrow transplantation 3 months later (autopsy not done).

\section{Discussion}

Aspergillus is a ubiquitous fungus present in the air, and its inhalation is extremely common. The most frequent manifestation of pulmonary aspergillosis is the saprophytic form, when fungus colonizes and proliferates in a preexisting cavity. ${ }^{7}$ However, in immunocompromised patients aspergillosis behaves like an opportunistic infection, giving rise to IPA. ${ }^{8}$ As already reported by others, in our group of immunocompromised patients the risk of IPA was $5 \%$. ${ }^{9,10}$ The presence of construction activity contiguous to a department of hematology is a widely recognized risk factor for IPA, ${ }^{11}$ and the risk of 
development of IPA also increases progressively with the number of granulocytopenic days, reaching a plateau of $70 \%$ among patients with granulocytopenia for more than 34 days. $^{12}$ In our group of patients, the mean duration of granulocytopenia was 33 days.

The prognosis of IPA in patients with leukemia remains poor. The mortality rate reaches an average of $50 \%$ to $60 \%$ when IPA occurs during chemotherapy-induced neutropenia and can exceed $90 \%$ in the setting of bone marrow transplantation. ${ }^{6}$ The prerequisites for improving prognosis include early recognition of IPA, shortening the neutropenic period with the use of hematopoietic growth factors, effective antifungal treatment, ${ }^{13}$ and surgical resection of localized IPA. ${ }^{14-17}$

The definitive diagnosis of IPA must be made by histologic studies. Bronchopulmonary necrosis, parenchymal and blood vessel invasion by fungal hyphae with ensuing thrombosis, infarction, and acute inflammation define IPA. Cavities occur by separation of the infarcted lung from adjacent viable lung, resulting in an intracavitary sequestrum of necrotic lung tissue. ${ }^{18}$ The roentgenologic correlation of this sequence of events is the air crescent sign. ${ }^{19}$ Unlike classic aspergilloma, in which the cavity preexists the fungal invasion, in IPA the proposed pathogenesis of cavity formation invokes destruction of lung parenchyma by phagocyte-derived proteolytic enzymes at the periphery of the infarct. ${ }^{20}$

Severe thrombocytopenia in patients with acute leukemia who undergo chemotherapy usually prevents biopsy of any tissue. Thus establishing a definitive diagnosis (i.e., histologic) in the absence of highly sensitive serologic tests is usually not possible before the surgical resection. Careful work from the early 1980s showed that a positive BAL culture during neutropenia $(<500 / \mu \mathrm{l})$ has a $90 \%$ positive predictive value for IPA. ${ }^{21,22}$ Of the eight BAL cultures considered to be positive, one $(12.5 \%)$ has been retrospectively classified in the NIPA group, and this margin of error does not differ from those in previous studies. In that case, the pathologist diagnosed an intrabronchial aspergilloma. The real problem is not the false positivity, however, but rather the false negativity of BAL. Of the 10 patients with negative BAL cultures, five had histologically proved IPA.

IPA is common enough to be considered early in the course of neutropenic patients. The presence of neutropenia, pneumonia, persistent fever despite broad-spectrum antibiotic therapy, hemoptysis, and an air crescent sign on the CT scan are the main criteria to suggest IPA in immunocompromised patients. Because of the severity of the prognosis, when the BAL culture remained negative, we considered the diagnosis of IPA to be highly likely when at least three of those five criteria were present. Of the 10 patients with negative BAL cultures, five had histologically proved IPA and five did not. In this group of 10 patients, seven met three criteria, one met four criteria, and two met all five criteria. When all five criteria were present the diagnosis of IPA was never incorrect. Among the total group of 18 patients, chest pain was the single discriminative sign between the IPA and the NIPA groups $(16.6 \%$ in the NIPA group vs $66.6 \%$ in the IPA group) ( $p<$ $0.05)$, and that could be explained by the pleural inflammation that is present in the IPA group. Nevertheless, the prognosis of IPA relapse is so poor that when clinical criteria suggestive of IPA are present, it is difficult to exclude surgery merely because the patient has no chest pain. CT scan could help to improve the diagnosis. Like others, we believe the air crescent sign is an insensitive marker for lung cavitation in IPA, ${ }^{18}$ appearing long after the onset of the disease. However, another CT sign, the halo sign, appears earlier during the evolution of IPA. ${ }^{23}$ In a recent report, at least one halo sign was present in more than $90 \%$ of patients with IPA, and the sign was very indicative of IPA in neutropenic patients. ${ }^{24}$ This CT sign must be given more attention in the future.

At present, clinicians have two agents that are therapeutically useful for invasive aspergillosis: amphotericin $\mathrm{B}$ and itraconazole. ${ }^{6}$ However, antifungal treatment must be given for long periods, for example, 6 months after hematologic recovery. ${ }^{24}$ Despite a well-conducted treatment, two severe complications have been observed. First, severe hemoptysis is common after apparently successful antifungal treatment. ${ }^{25}$ Second, for patients with hematologic malignant diseases, additional cytotoxic chemotherapy is often necessary to achieve or maintain the response or remission of the underlying disease before the antifungal treatment has been completed. The rate of IPA relapse during subsequent neutropenia is high, even after large doses of amphotericin $\mathrm{B}$, because persistent intracavitary fungal growth may serve as a reservoir for recurrent fungal invasion in patients with prolonged neutropenia or a repeated episode of severe neutropenia. ${ }^{26}$ In two patients, surgery was performed on an urgent basis because the mass was located close to the main 
pulmonary artery and the risk of massive hemoptysis was high. Early surgical resection seems effective in preventing life-threatening hemoptysis. ${ }^{15,16,24,27}$ The risk of hemoptysis is higher after recovery of granulocytes, ${ }^{25}$ but fatal bleeding can occur more than 6 weeks after the beginning of amphotericin B therapy or hematologic recovery. ${ }^{28}$ Surgery also seems effective in preventing IPA recurrence, with one documented case of IPA relapse among 54 $(1.8 \%)$ other cases already reported in the literature. ${ }^{15-17,24}$ In our data, one patient died of what we suspected to be invasive cerebral aspergillosis during a bone marrow transplantation 3 months after the surgical resection of a localized IPA. Other modalities (intravenous amphotericin B or oral itraconazole) for prevention of IPA reactivation can be discussed. ${ }^{29,30}$ However, the mandatory rapid reinstitution of cytotoxic treatment for the underlying hematologic disease implies a new period of neutropenia before the antifungal treatment is completed, and few patients with persistent neutropenia and invasive aspergillosis survive. ${ }^{6}$ Moreover, a small number of patients with persistent neutropenia have responded to liposomal amphotericin ${ }^{31}$ or itraconazole $^{32}$ during neutropenia, but resolution of aspergillosis has followed neutrophil recovery. In patients with localized IPA and no contraindication to surgery, the risks of the surgical resection are limited (no complications in our patients). Finally, the benefit of surgery is rapid treatment of areas of infection, which allows for rapid retreatment with protocols including high-dose chemotherapy and prevention of life-threatening hemoptysis.

As in other series, ${ }^{15,16}$ the most common procedure performed was a lobectomy without any complications or deaths. This contrasts strongly with the treatment program for patients with classic aspergilloma. However, young age of the patient, good function and good reexpansion of the residual lung, and noninduration of the hilar structure by tuberculosis are the rule in localized IPA. ${ }^{16}$ Moreover, surgery was always performed after the end of neutropenia, and only two patients required platelet transfusion before surgery. That explains the absence of bleeding and infectious complications. Because the recovery must be rapid without any prolonged air leak to permit treatment of the underlying hematologic disease, and also because the disease is often more diffuse inside the lobe than the CT scan indicates, we usually performed lobectomies rather than wedge resections. We have not used video-assisted surgery. Pleural adhesions, in- flammation of the hilar structure, and location of the lesion close to the branches of the pulmonary artery or even the main pulmonary artery itself are not favorable conditions for minimally invasive surgery.

In conclusion, a poor outcome after hemoptysis and IPA relapses is obvious. ${ }^{6}$ Surgery for these indications appears safe (rapid recovery, no complications, no deaths). Therefore we advocate that surgical therapy must be performed as soon as the diagnosis of localized IPA is suggested by clinical and radiologic criteria, even if not confirmed by histologic studies.

\section{REFERENCES}

1. Winson DJ, Gall RP, Meyer DV, Young LS, UCLA Bone Marrow Transplantation Group. Infectious complications of human bone marrow transplantation. Medicine 1979;58:1-31.

2. Meyers JD. Infections in bone marrow transplant recipients. Am J Med 1986;81(Suppl 1A):27-38.

3. Krowka MJ, Rosenov EC III, Hoagland HC. Pulmonary complications of bone marrow transplantation. Chest 1985; 87:237-46.

4. Meyer RD, Young LS, Armstrong D, Yu B. Aspergillosis complicating neoplastic disease. Am J Med 1973;54:6-15.

5. Bodey G, Bueltmann B, Duguid W, Gibb D, Hanak H, Hotchi M, et al. Fungal infections in cancer patients: an international autopsy survey. Eur J Clin Microbiol Infect Dis 1992;11:99-109.

6. Denning DW, Stevens DA. Antifungal and surgical treatment of invasive aspergillosis: review of 2121 published cases. Rev Infect Dis 1990;12:1147-201.

7. Suen HC, Wright CD, Mathison DJ. Surgical management of pulmonary aspergillosis. Surg Clin North Am 1993;4:671-81.

8. Hinson KFW, Moon AJ, Plummer NS. Bronchopulmonary aspergillosis: review and report of eight cases. Thorax 1952; 7:317-33.

9. Cordonnier C, Bernaudin JF, Bierling P, Huet Y, Vernant JP. Pulmonary complications occurring after allogenic bone marrow transplantation: a study of 130 consecutive transplanted patients. Cancer 1986;58:1047-54.

10. Pannuti CS. Nosocomial pneumonia in adult patients undergoing bone marrow transplantation. J Clin Oncol 1991;9:7784.

11. Weems JJ, Davis BJ, Tablan OC, Kaufman L, Martone WJ. Construction activity: an independent risk factor for invasive aspergillosis and zygomycosis in patients with hematologic malignancy. Infect Control 1987;8:71-5.

12. Gerson SL, Talbot GH, Hurwitz S, Strom BL, Lusk EJ, Cassileth PA. Prolonged granulocytopenia: the major risk factor for invasive pulmonary aspergillosis in patients with acute leukemia. Ann Intern Med 1984;100:345-51.

13. Fisher BD, Armstrong D, Yu B, Gold JWM. Invasive aspergillosis: progress in early diagnosis and treatment. Am J Med 1981;71:571-7.

14. Moreau P, Zahar JR, Milpied N, Baron O, Mahé B, Wu D, et al. Localized invasive pulmonary aspergillosis in patients with neutropenia: effectiveness of surgical resection. Cancer 1993;72:3223-6. 
15. Wong K, Waters CM, Walesby RK. Surgical management of invasive pulmonary aspergillosis in immunocompromised patients. Eur J Cardiothorac Surg 1992;6:138-43.

16. Young VK, Maghur HA, Luke DA, McGovern EM. Operation for cavitating invasive pulmonary aspergillosis in immunocompromised patients. Ann Thorac Surg 1992;53:621-4.

17. Robinson LA, Reed EC, Galbraith TA, Alonso A, Moulton A, Fleming WH. Pulmonary resection for invasive Aspergillus infections in immunocompromised patients. J Thorac Cardiovasc Surg 1995;109:1182-97.

18. Pai U, Blinckhorn RJ Jr, Tomascheski JF Jr. Invasive cavitary pulmonary aspergillosis in patients with cancer: a clinicopathologic study. Hum Pathol 1994;25:293-303.

19. Curtis AM, Smith GJW, Ravin CE. Air crescent sign of invasive aspergillosis. Radiology 1979;133:17-21.

20. Przyjemski C, Mattii R. The formation of pulmonary mycetoma. Cancer 1980;46:1701-4.

21. Barnes AJ, Denning DW. Aspergillus: significance as a pathogen. Rev Med Microbiol 1993;4:176-80.

22. Saito H, Anaissie EJ, Morice RC, Dekmezian R, Bodey GP. Bronchoalveolar lavage in the diagnosis of pulmonary infiltrates in patient with acute leukemia. Chest 1988;94: 745-9.

23. Kuhlman JE, Fishman EK, Burch A, Karp JE, Zerhouni EA, Siegelman SS. CT of invasive pulmonary aspergillosis. AJR Am J Roentgenol 1988;150:1015-20.

24. Caillot D, Casanovas O, Bernard A, Couaillier JF, Durand C, Cuisenier B, et al. Improved management of invasive pulmonary aspergillosis in neutropenic patients using early thoracic computed tomographic scan and surgery. J Clin Oncol 1997; 15:139-47.

25. Abelda SM, Talbot GH, Gerson SL, Miller WT, Cassileth PA. Pulmonary cavitation and massive hemoptysis in invasive pulmonary aspergillosis: influence of bone marrow recovery in patients with acute leukemia. Am Rev Respir Dis 1985; 131:115-20.

26. Robertson MJ, Larson RA. Recurrent fungal pneumonias in patients with acute nonlymphocytic leukemia undergoing multiple courses of intensive chemotherapy. Am J Med 1988;84:233-9.

27. Kibbler CC, Milkins SR, Bahmra A, Spiteri MA, Noone P, Prentice HG. Apparent pulmonary mycetoma following invasive pulmonary aspergillosis in neutropenic patients. Thorax 1988;43:108-12.

28. Aisner J, Schimpff SC, Wiernick PH. Treatment of invasive aspergillosis: relation of early diagnosis and treatment to response. Ann Intern Med 1977;86:539-43.

29. Karp JE, Burch PA, Merz WG. An approach to intensive antileukemia therapy in patients with previous invasive aspergillosis. Am J Med 1988;85:203-6.

30. Harousseau JL, Milpied N, Morin O, Germaud P, Legent F. Bone marrow transplantation in patients with preexisting aspergillosis [abstract]. Bone Marrow Transplant 1986; 1(Suppl 1):50.

31. Chopra R, Fidding A, Goldstone AH. Successful treatment of fungal infections in neutropenic patients with liposomal amphotericin (AmBisome): a report of 40 cases from a single centre. Leuk Lymphoma 1992;97:135-44.

32. Denning DW, Lee JY, Hostetler JS, et al. NIAID Mycoses Study Group multicenter trial of oral itraconazole therapy of invasive aspergillosis. Am J Med 1994;97:135-44.

\section{Discussion}

Dr. Marvin Pomerantz (Denver, Colo.). These authors operated on 18 patients with presumed IPA without a death or a complication. This is certainly a monumental task. As they pointed out, only 12 of these 18 patients did have IPA, but one had an aspergilloma and the other four had other pulmonary infections. However, I believe the title of their paper gives the impression that they are operating on patients during a neutropenic phase, when, actually, at the time of the operation, the polymorphonuclear cell count was over $6000 / \mu \mathrm{l}$ and the platelet count was almost $250,000 / \mu$ l. They also had the luxury of having their patients receive antibiotic therapy for an average of 32 days.

I have only one question for the authors. They reported having 80 patients with presumed pulmonary aspergillosis, and they operated on 18 . What happened to the other 62 patients?

Dr. Lary A. Robinson (Tampa, Fla.). Three years ago we presented our series of 16 immunocompromised patients with IPA who underwent major pulmonary resections. Most of them had undergone bone marrow transplantation. We agree that aggressive surgical management of this problem offers their only chance of survival after bone marrow transplantation. In the literature, the mortality from IPA in this group after medical treatment is $95 \%$ and up.

I would like to make several comments. The diagnosis is often hard to make before the operation. Bronchoscopic examinations and needle biopsies are not necessary and did not help us to make the diagnosis.

One important radiographic sign is the halo sign. Most IPAs will have a halo on chest CT scan if they are discovered early in the course of the infection and if the patient is neutropenic. When the white cell counts recover, however, the white cells apparently are able to phagocytize the necrotic material in the center of the Aspergillus mass. Then they end up having a circle of dense material around the cavitary abscess, which radiographically on CT scan is the air crescent sign. These two signs were quite diagnostic for IPA, at least in our hands. Incidentally, most of our patients were operated on when they had very few white cells, usually fewer than 500 white cells, and the halo sign was very common.

Initially in our experience we waited for a while before operating, but the patients then became quite sick. This delay in operation led to surgery on very sick patients and some subsequent deaths. We have changed to early operation on these patients, most of the time while they are neutropenic. We did not have any pleural space problems, pleural infections, or problems with bleeding in these neutropenic patients. Our best results were with early operation.

The deaths that we had were in allogeneic patients undergoing bone marrow transplantation. We had five such patients, and three of them died of diffuse alveolar hemorrhage 7 to 10 days after the operation. The one patient who required a ventilator before the operation also died after the operation.

I would like to ask the authors one question: Have you had any experience with IPA in patients who have had bone marrow transplantation, and were any of those 
allogeneic transplant patients? That group had the highest mortality in our hands. Otherwise, the other patients did extremely well.

Dr. Jhingook Kim (Seoul, Korea). Because the complicated aspergillosis region is not affected by the malignant disease, it is not necessary to resect a margin of tissue. I try to save as much of the normal lung as possible. Therefore, I prefer a retroresection or a precise excision of the lesion after adequate and sufficient use of amphotericin.

If the lesion is in the middle of the upper lobe, do you still prefer to do a right upper lobectomy rather than just a precise excision of the region of the aspergillosis?

Dr. Khaled M. Al-Kattan (Riyadh, Saudi Arabia). I have two questions. First, some of these lesions are multiple, and even if they have caused hemoptysis, it can be difficult to tell which one is responsible, especially after bone marrow transplantation. What do you do with these?

Second, some of those lesions are small, and the oncologist or the infectious disease group has fairly good success eliminating them with high doses of amphotericin. How do you know when to operate? How do you decide that the medical treatment is a failure or should not be tried?

Dr. Baron. Thank you for your comments. Dr. Pomerantz, the 62 patients with IPA who were not treated surgically had more diffuse disease, with bilateral lesions, which contraindicated surgical resection.

We use the term neutropenia because the disease always developed in patients during a neutropenic period caused by chemotherapy for hematologic disease. However, except when the risk of hemoptysis was too high, and that occurred two times in our series, we did not operate on those patients before the end of neutropenia.

To respond to your comment about the use of early antifungal treatment and the use of hematopoietic growth factors, the mass does not grow fast enough to force us to operate before the end of neutropenia. Usually our main surgical indication was to prevent an IPA relapse during the subsequent period of neutropenia mandatory to treat the underlying disease. We are aware from other reports that localized forms of IPA have been resected successfully during the neutropenic period, but so far that is not our policy.

Dr. Robinson, you emphasized the halo sign. In the future we will have to pay more attention to this CT sign because it appears earlier during the evolution of the disease than does the air crescent sign. We have not operated on those kinds of patients during or after a bone marrow transplantation. All 18 patients were operated on after the initial program of chemotherapy for hematologic disease.

Dr. Kim inquired about the advisibility of using wedge resection or lobectomy. We think that the disease is often more diffuse in the lobe than we can tell on the CT scans, so we advocate wide resection. However, when the nodule is a very peripheral nodule, we have done wedge resection.

Dr. Kim. Let me clarify my question. When the region is deep inside the lung parenchyma, too deep for wedge resection, do you prefer lobectomy or do you just open the lung and take out the dead region precisely?

Dr. Baron. Usually we have done a lobectomy.

Dr. Deslauriers. The other question concerned the timing of operation versus continuing medical treatment. When do you decide that?

Dr. Baron. The problem with those patients is the context, that context being their hematologic disease. We cannot wait 3 months to treat the IPA, and we need at least 3 months of effective antifungal treatment to cure the IPA. We operate as soon as the neutropenia period is completed, but we never have operated before the completion of the neutropenia, because we wanted to avoid any complication of the neutropenic period. 